\title{
Optimal Placement of Sensors in Mission-specific Mobile Sensor Networks
}

\author{
Hicham Ouchitachen ${ }^{1,{ }^{*}}$, Abdellatif Hair ${ }^{2}$, and Najlae Idrissi ${ }^{3}$ \\ Sultan Moulay Slimane University, Faculty of Sciences and Techniques \\ ${ }^{1,2}$ Applied Mathematics and Scientific Calculus Laboratory \\ ${ }^{3}$ Information Processing Decision Support Laboratory \\ P.B. 523, Beni-Mellal, Morocco. \\ *h.ouchitachen@gmail.com
}

\begin{abstract}
Placement of nodes has been the major challenge in wireless sensor networks. The data reported from a sensor is only useful when the position of that sensor is found. In this context, several techniques have been proposed to conserve power consumption and to prolong the lifetime of the wireless sensor networks. In this paper, we propose a new algorithm that accurately finds the best locations of sensors while minimizing the average energy consumed in the network. More precisely, we consider a critical network in each sensor satisfying its own missions and depending on its locations. In addition to fulfill their mission, the sensor tries to maintain a good neighboring nodes quality. we determine the location of node by using two criteria: the cost and the quality of communication. The aim of this work is to develop a new algorithm so as to solve the complicated optimization problem posed in this case while minimize the total energy consumption. Our simulation results demonstrate that our algorithm is very advantageous in terms of convergence to the appropriate locations.
\end{abstract}

Keywords: energy, node placement, sensor networks, communication quality, genetic algorithms

Copyright (C) 2015 Institute of Advanced Engineering and Science. All rights reserved.

\section{Introduction}

Wireless Sensor Networks (WSNs) [1, 2] are forecast to become highly integrated to our daily activities [3]. The WSNs are becoming increasingly popular for monitoring spatial phenomena. Indeed, they are deployed to collect data from the environment, process sensed data and take action accordingly. Typical applications of the WSNs include environmental control such as fire fighting or marine ground erosion, but also sensors installation on bridges or buildings to monitor earthquake vibration patterns and various surveillance tasks such as intruder surveillance on premises. Yet, it is still very early in the lifetime of such systems and many research challenges exist, for instance their computational capabilities, limited storage, and especially their dependence on a limited power supplied by a battery. The low power consumption is one of the major challenges that face WSNs today.

Nodes of both sensor networks and conventional networks such as wireless LANs and cellular networks, focus on providing a good communication quality, or getting letter quality services, except that the nodes of the sensor networks are, moreover, interested in the satisfaction of their missions. Thus we call those kinds of networks, critical networks, where the quality and satisfaction of the mission of each node is very important to improve the overall performance of critical networks mission. Monitoring in mission-critical environment with the deployment of WSNs is one of the most widely-used application areas. Mission-critical environment implies monitoring tasks that happened in locations which are difficult to get access to or easy to lead destroy of the network deployment: scenarios in hazard and emergency monitoring situations such as chemical pollution, environment-oriented pollutions and land security. In the near future it can be expected that surveillance areas will be equipped with a range of smart sensors to provide parts of overall event detection and service. Hence, in this class of networks, it is important to control the mobility of a node by jointly considering its mission and communication quality, so as to improve the overall performance, by controlling the mobility problem which is much difficult to study currently. 
Researchers have conducted investigations on WSNs at almost every layer of the communication protocol stack. There exist several papers on efficient routing algorithms and data aggregation techniques $[4,5]$, localization techniques $[6,7]$ and medium access control (MAC) methods $[8,9]$. In addition, researchers take advantage of the flexibility of WSNs by designing protocols that positively affect energy consumption. The majority of researcher papers that appeared until now consider sensor network to be static.

Recently, mobility control or node placement problems become the aim of researches and studies in mobile sensor and ad hoc networks. In [10] and [11], the modeling transmission power consumption was considered as a tool of studying problems of relay node placements related to minimizing power consumption or maximizing network lifetime, via a function of the distance between two nodes. In [12] and [13], a study of problems that place minimum number of relay nodes as long as the constraints on node connectivity or traffic demands are satisfied. In [14], a mobility control problem in ad hoc networks is studied to maximize the throughput based on the IEEE 802.11 throughput analysis in [15] without considering the variation of the link capacity according the distance change between two end nodes. In [16, 18], problems that place relay nodes considering the throughput of the network are studied. In [16], considering the probabilistic model for the positions of nodes, an algorithm for the relay node placement that maximizes the throughput of the network is proposed. In [17], joint relay node placement and assignment problem is studied. Once the relay node assignment is determined, the problem in [17] is reduced to the problem of finding the optimal position of a single relay node while other nodes that communicate with the relay node are assumed to be fixed. In [18], a cascaded network in which relay nodes form a single chained communication flow is considered and a gradientbased algorithm is proposed to control the position of relay nodes to maximize the minimum throughput among those of all the links in the chain.

The work that we consider in this paper is clearly different in several critical aspects. First, we consider a network with multiple nodes with controllable mobility in a mesh topology in a two-dimensional space. In fact, the extension from a single node with controllable mobility $[16,17,19,20]$ or from the chained topology $[18,21]$ to multiple nodes with controllable mobility in a mesh topology is not straightforward.

The rest of the paper is organized as follows. Section 2 deals with mathematical modeling of the problem. Section 3 describes numerical results. Conlusion is presented in section 4.

\section{Research Method}

\subsection{Model network}

In this section, we present our approache to solve the problem of optimal placement of sensors in mobile wireless sensors network. We consider a WSNs (Fig.1) containing several sensors managed by a base station (BS). The nodes satisfy their missions depending on their locations. To accomplish their mission, the sensor nodes have to maintain a good quality of communication with their neighbors, which also depends on their locations. Our objective is to find the best locations of sensor nodes by developing Sensor's Genetic Algorithm (SGA).

The choice of Genetic Algorithms (GA) in this work is motivated firstly by finding an algorithm that permits to solve the non-convex optimization problems; GA impose no regularity on the function studied (continuity, differentiability, convexity ...). Secondly by getting a robust algorithm for looking for a solution very close to optimal or near-optimalsolution.

Thus, in order to solve the energetic constraint which is the main challenge of WSNs, the optimization problem to be solved is formulated as follows :

We take $n$ sensors caracterized each one by two cost functions: Mission and communication costs. We then aim to minimize the total cost by choosing the best location of each sensor calculated via SGA.

We suppose that a set of $n$ sensors is deployed in a geographic area of interest to supervise a given physical phenomenon. The topology of a WSNs is represented by the graph $G=(C, E)$, where $C=\{1,2, \ldots, n\}$ is a set of $n$ sensors and $E \subset C \times C$ is the set of wireless links between the various sensors. We denote $C_{v(i)}$ the neighbor set of the sensor $i$. In the below, we present the meanings of the notations used in our modeling. 


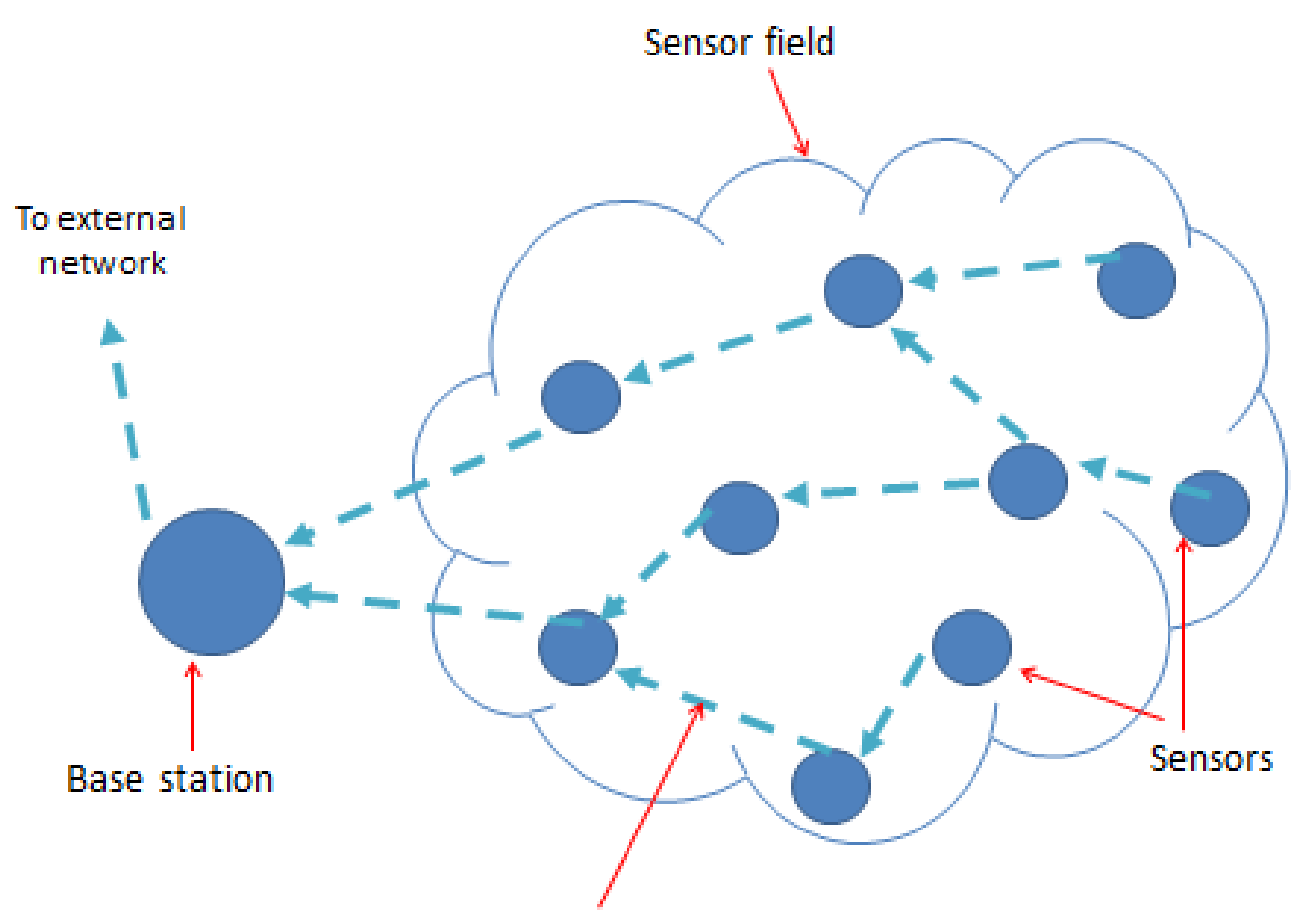

Wireless communication link

Figure 1. WSN architecture

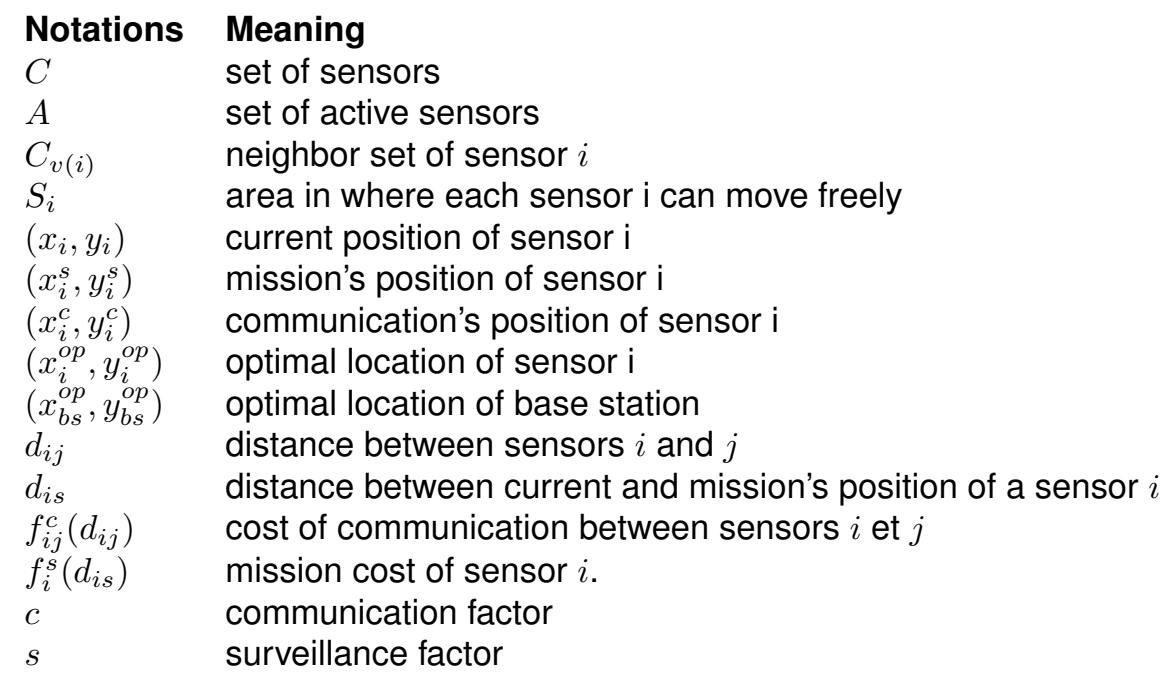

\subsection{Optimal placement of sensors}

To minimize the energy consumed by all sensors considering the joint communication and mission costs, we propose to find the optimal location $\left(x_{i}^{o p}, y_{i}^{o p}\right)$ of each sensor by solving the following optimization problem [22]:

$$
\min f(x, y)=\Sigma_{i} s f_{i}^{s}\left(d_{i s}\right)+\Sigma_{i} \Sigma_{j \in C_{v(i)}} c f_{i j}^{c}\left(d_{i j}\right)
$$




$$
\text { subject of }\left(x_{i}, y_{i}\right) \in S_{i} \quad \forall(i, j) \in C \times C_{v(i)}
$$

We put:

$$
\begin{gathered}
f_{i}^{c}\left(d_{i j}\right)=\Sigma_{j \in C_{v(i)}} f_{i j}^{c}\left(d_{i j}\right) \\
V=\left(x_{1}, y_{1}, x_{2}, y_{2}, \ldots, x_{n}, y_{n}\right) \\
F_{s}(x, y)=\Sigma_{i} f_{i}^{s}\left(d_{i s}\right) \\
F_{c}(x, y)=\Sigma_{i} f_{i}^{c}\left(d_{i j}\right) \\
F(V)=f(x, y)
\end{gathered}
$$

So (1) becomes:

$$
\begin{gathered}
\min F(V)=s F_{s}(V)+c F_{c}(V) \\
\text { subject of } V \in \prod_{i=1}^{n} S_{i}
\end{gathered}
$$

\section{Proposed algorithm}

To solve the optimization problem given by (7), we implement the following algorithm:

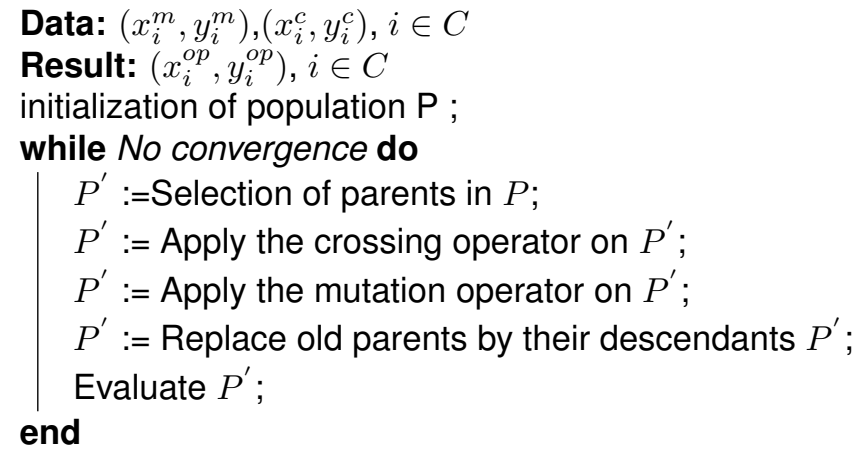

Algorithm 1: Algorithm SGA

Our algorithm starts by generating an initial population $P$ and evaluating the adaptation of all individuals in initial population. Then the individuals are randomly selected for reproduction according to the principle of survival of the fittest. After that the children (or descendants) are generated applying the following two genetic operators: crossover and mutation. Those children are moved to a new population $P^{\prime}$ and will be replaced, in whole or in part, by the children of previous generations. The new population of individuals will then take over from one generation to the next, each representing a generation iteration until reaching the stopping criterion.

\subsection{Optimal placement of base station}

\section{Result and Analysis}

In this section, we provide numerical results given by our algorithm SGA used to find the best locations of sensors. We set cost functions and parameters as follows [22]:

$f_{i j}^{s}\left(d_{i s}\right)=5 \exp \left(10^{-2} d_{i s}-1\right), f_{i j}^{c}\left(d_{i j}\right)=100 \exp \left(\frac{-10}{12} \log _{2}\left(10^{6}\left(d_{i j}\right)\right)\right)$, and $C=\{1,2, \ldots, 12\}$. We consider a network in Fig.2, which consists of 12 nodes, where $\mathrm{MP}=\left(x_{i}^{s}, y_{i}^{s}\right)$ and $\mathrm{CP}=\left(x_{i}^{c}, y_{i}^{c}\right)$ denotes respectively the mission and communication's position of each sensor $i$. 


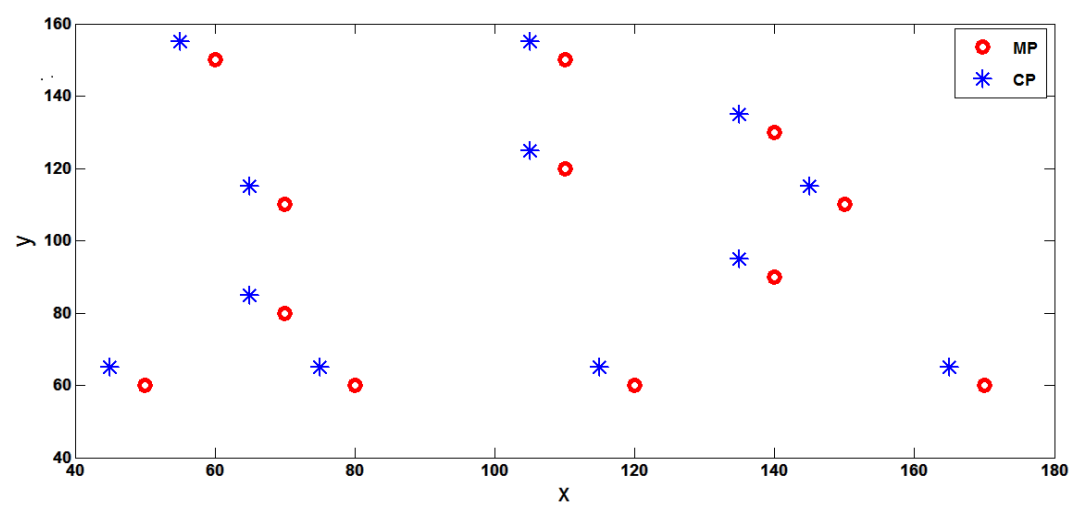

Figure 2. Simulation topology

Figure 3 and Figure 4 illustrate that as the surveillance factor becomes larger, as each node gets closer to its target location in order to reduce the cost of its mission, as the communication quality cost increases. On the other hand, as the communication factor becomes larger, as each node gets closer to each other to reduce the communication cost, proceeding the increased mission cost (surveillance).

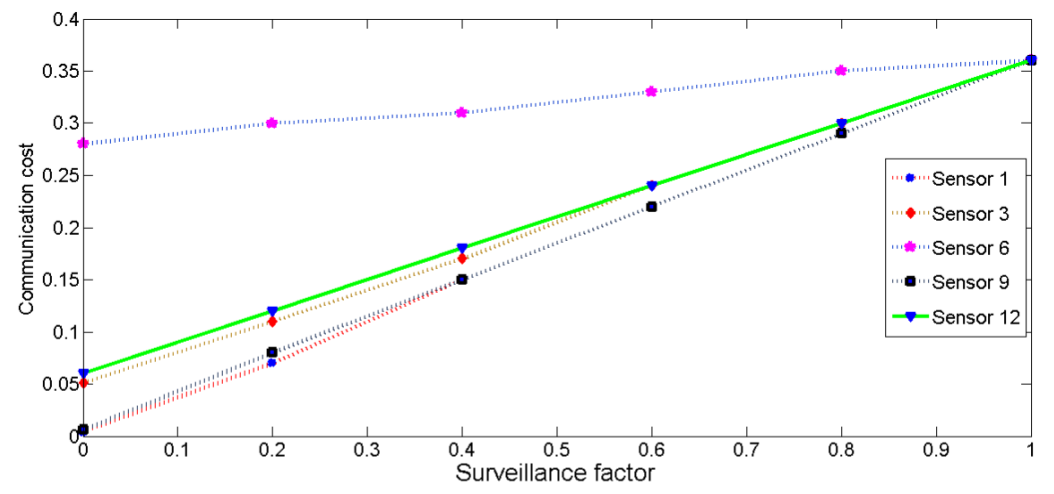

Figure 3. Variation of communication cost

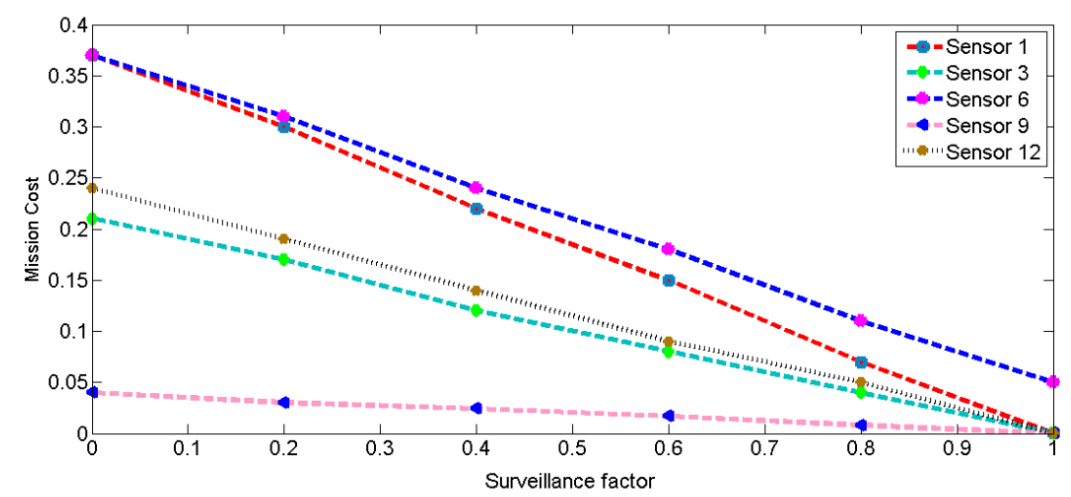

Figure 4. Variation of mission cost 


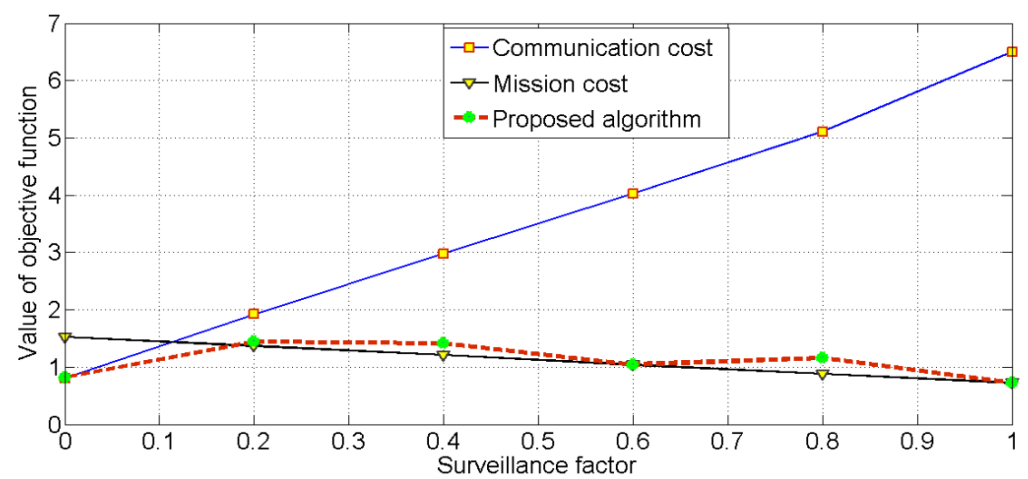

Figure 5. Performances of our algorithm

Figure 5 illustrate the comparaison of two cases with our proposed algorithm which are as follows:

First case: each node updates its location considering only its mission(surveillance).

Second case: each node updates its location considering only its communication quality.

The best locations of sensors and the base station calculated respectively by using two algorithms SGA and BGA are represented by the Figure 6 .

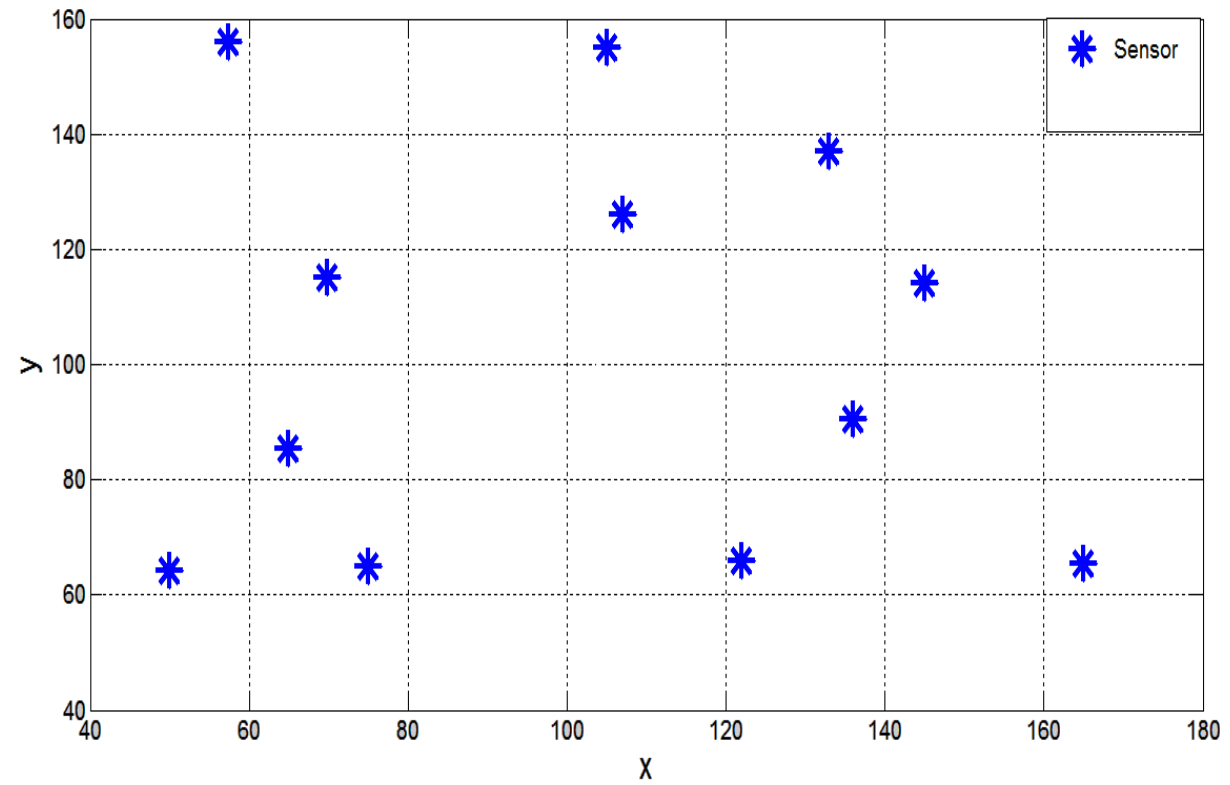

Figure 6. Best locations of sensors given by SGA

Figure 7 shows clearly that, comparing to the Simulated Annealing (SA), our node placement algorithm SGA can provide the appropriate location of each node according to the variation of weight factors while minimizing the total weighted cost for missions and communication qualities of all nodes. Our algorithm SGA is very advantageous in terms of convergence, this fact is shown clearly in Figure 8. 


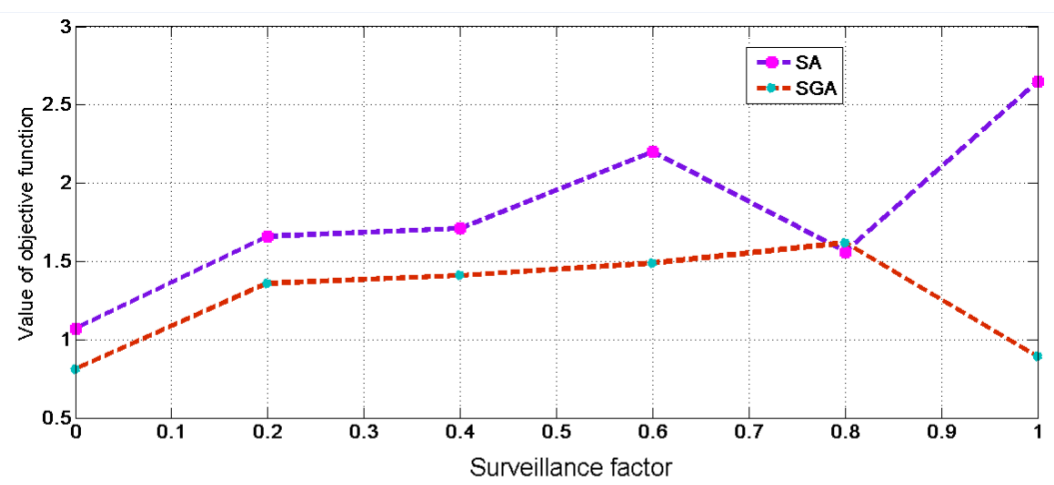

Figure 7. Convergence of objective function given by SGA

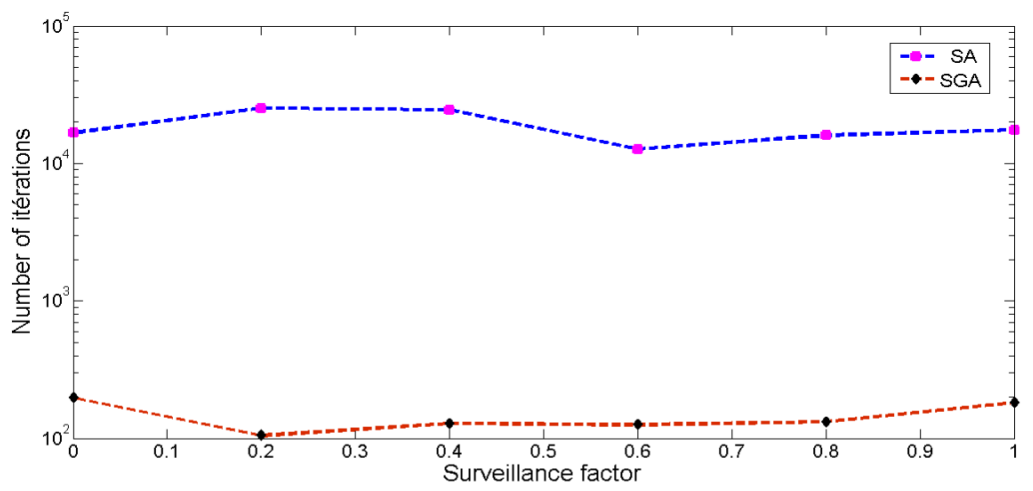

Figure 8. Number of iteration as function of mission factor given by SGA

\section{Conclusion}

In this paper, we proposed a novel algorithm to solve the optimization problem formulated in order to find the optimal locations of sensors in mobile wireless sensor networks, where each node tries to minimize the weighted sum of mission and communication cost in a distributed way. Our approach is based on genetic algorithms. We show that, comparing to other techniques, our algorithm is very advantageous in terms of convergence to the optimal solution. Numerical results prove that our algorithm provides the appropriate location of each node by considering jointly the cost of mission and the quality of communication.

\section{References}

[1] I. Akyildiz, T. Melodia, and K. Chowdhury, "A survey on wireless multimedia sensor networks," Computer networks, vol. 51, no. 4, pp. 921-960, 2007.

[2] I. Almalkawi, M. Guerrero Zapata, J. Al-Karaki, and J. Morillo-Pozo, "Wireless multimedia sensor networks: Current trends and future directions," Sensors, vol. 10, no. 7, pp. 6662-6717, 2010.

[3] K. Fowler, "The future of sensors and sensor networks survey results projecting the next 5 years," in Proc. Sensors Applications Symposium, 2009 (SAS 2009), New Orleans, LA, USA, Feb 2009, pp. 1-6.

[4] J.N. Al-Karaki, R. Ul-Mustafa, A.E. Kamal, "Data aggregation and routing in Wireless Sensor Networks: Optimal and heuristic algorithms," Computer Networks 53, 2009, pp. 945-960. 
[5] S. Zarifzadeh, A. Nayyeri, N. Yazdani, A. Khonsari, H.H. Bazzaz, "Joint range assignment and routing to conserve energy in wireless ad hoc networks," Computer Networks 53,2009 , pp. 1812-1829.

[6] G. Mao, B. Fidan, B.D.O. Anderson, "Wireless sensor network localization techniques," Computer Networks 51, 2007, pp. 2529-2553.

[7] K. Yedavalli, B. Krishnamachari, "Sequence-Based Localization in Wireless Sensor Networks," Mobile Computing, IEEE Transactions on 7 (2008), pp. 81-94.

[8] I. Demirkol, C. Ersoy, F. Alagz, "MAC protocols for wireless sensor networks: A survey," IEEE Communications Magazine 44, 2006 pp.115- 121.

[9] H. Tseng, A. Pang, J. Chen, C. Kuo, "An adaptive contention control strategy for IEEE 802.15.4-based wireless sensor networks," IEEE Transactions on Vehicular Technology 58, 2009, pp. 5164-5173

[10] J. Pan, Y.T. Hou, L. Cai, Y. Shi, S.X. Shen, "Topology control for wireless sensor networks," in ACM Mobicom , 2003, pp. 286-299.

[11] E.R. Chittimalla, A. Venkateswaran, V. Sarangan, R. Acharya, "On the use of nodes with controllable mobility for conserving power in manets," in IEEE ICDCSW, 2006, pp. 88-93.

[12] S. Misra, S.D. Hong, G. Xue, J. Tang, "Constrained relay node placement in wireless sensor networks to meet connectivity and survivability requirements," in IEEE INFOCOM, 2008, pp. 281-285.

[13] B. Lin, P.-H. Ho, L.L. Xie, X. Shen, "Relay station placement in IEEE 802.16 j dual-relay mmr networks," in IEEE ICC , 2008, pp. 3437- 3441.

[14] T. Nadeem, S. Parthasarathy, "Mobility control for throughput maximization in ad hoc networks," Wireless Communications and Mobile Computing 6 (7) , 2006, pp. 951-967.

[15] G. Bianchi, "Performance analysis of the IEEE 802.11 distributed coordinated function," IEEE Journal on Selected Areas in Communications 18 (3), 2000, pp. 535-547.

[16] A. So, B. Liang, "Enhancing WLAN capacity by strategic placement of tetherless relay points," IEEE Transactions on Mobile Computing 6 (5), 2007, pp. 522-535.

[17] A. Srinivas, E. Modiano, "Joint node placment and assignment for throughput optimization in mobile backbone networks," in IEEE INFOCOM , 2008, pp. 1804-1812.

[18] C. Dixon, E.W. Frew, "Maintaining optimal communication chains in robotic sensor networks using mobility control," Mobile Networks and Applicaitons 14 (3), 2009, pp. 281-291.

[19] H.-T. Roh, J.-W. Lee, "Optimal placement of a relay node with controllable mobility in wireless networks considering fairness," in IEEE CCNC, 2010.

[20] H.-T. Roh, J.-W. Lee, "Joint node scheduling and relay node placement in wireless networks with a relay node with controllable mobility," Wireless Communications and Mobile Computing 12 (8), 2012, pp. 699-712.

[21] H.-T. Roh, J.-W. Lee, "Communication-aware position control for mobile nodes in vehicular networks," IEEE Journal on Selected Areas in Communications 29 (1), 2011, pp. 173-186.

[22] H.-T. Roh, J.-W. Lee, "Joint Mission and Communication Aware Mobility Control in Mobile Ad-Hoc Networks," WiOpt'12: Modeling and Optimization in Mobile, Ad Hoc, and Wireless Networks, May 2012, Paderborn, Germany. pp.124-129. 